\title{
La satira notturna, tra frastuoni plebei e rime aristocratiche
}

\section{Marco Fincardi}

\section{Un tema imbarazzante}

Nella letteratura italiana, soprattutto quella in rima, sono rarissimi i riferimenti a un fragoroso rituale di derisione notturna - per secoli molto diffuso in Italia e nel resto d'Europa - che in toscano è usuale chiamare scampanata, in francese charivari, e in inglese skimmington, riding, oppure rough music. ${ }^{1}$ Non così infrequente è trovare testi che tocchino questo soggetto in alcune letterature europee: nella opere in rima britanniche si va da Andrew Marvell (Last Instructions to a Painter), a Samuel Butler (Hudibras) poi a Jonathan Swift (A Quiet Life, and a Good Name), per arrivare a Thomas Hardy (The Bride-Night Fire), ${ }^{2}$ senza sostanziale soluzione di continuità tra XVII e XIX secolo. E pure nel teatro colto inglese i riferimenti a scampanate sono ricorrenti. Per i poeti inglesi è abbastanza comune riferire degli skimmington fatti con fracasso di pentole a mariti o mogli che non abbiano assolto adeguatamente il proprio ruolo coniugale. In tali occasioni le rime poetiche si soffermano in particolare nelle descrizioni dei riding, con persone-oppure pupazzi che le raffigurano - messe a cavalcare a ritroso di asini o cavalli, tra rumori e scherni degli astanti. ${ }^{3}$ Nei versi dei poeti inglesi, oltretutto, in genere appare una compiaciuta affinità culturale degli autori col rituale popolare, senza che la cosa desti particolare scalpore tra i loro lettori. In Francia, tra XVII e XVIII secolo, alcuni drammaturghi della corte di Sceaux producono canzoni in rima per descrivere degli charivari, tra musiche e danze, a chiusura di commedie che rielaborano alcuni temi farseschi della commedia dell'arte e della commedia antica greca e latina. Così il teatro alla moda propone al proprio pubblico di principi e nobili commedie come Le charivari di Florent Carton D'Ancourt, nel 1697, e nel 1714 Le mariage de Ragonde et de Colin di Néricault Destouches, musicata da Jean-Joseph Mouret. ${ }^{4}$

Per trovare versi in lingua italiana come quelli di Samuel Butler che nel 1663 descrive dettagliatamente un 'Hideous clamour, and a loud one / As if all sorts of noise had been / Contracted into one loud din', occorre invece cercare nelle opere di autori minori o sconosciuti. ${ }^{5}$ Nelle rime in latino maccheronico del Baldus di Teofilo Folengo - pubblicato nel 1517 e poi riveduto in varie nuove redazioni 
fino al 1552 - si fa la baia pubblicamente a un contadinaccio arricchito che sta a capo del villaggio di Cipada, ma evitando di rendere palese l'aggressione comica collettiva verso questo vecchio vedovo attratto dalla bella e giovane Berta, sposa di un cavaliere furfante da lui fatto imprigionare: 'Sic adeo intantum schioppantur pectora risu, / Non pensat eos pro se grignare Tognazzus, / Imo plus cignat Bertae, cui Berta recignat: / Bertaque dat bertam, bertezans atque bretonans' ${ }^{6}$ Per burla, così, la donna irretisce Tognazzo al ballo, mantenendo però lo scherno in una celata forma ambigua, senza che si giunga apertamente alla scampanata. Nelle poesie satiriche di Pietro Aretino e Francesco Berni, nel XVI secolo, ci sono solo riferimenti molto vaghi a forme di derisione notturna codificate nella tradizione popolare.

Con la fine del XVI secolo, il concretizzarsi della Controriforma ha evidenti effetti nell'allontanare i letterati italiani - già molto inclini a ricavarsi spazi élitari - dai linguaggi comici più vicini al popolo. È soprattutto il cardinale Carlo Borromeo a indurre gli intellettuali a forme di autocensura sulle scampanate, ingiungendo la messa al bando di rituali profani irriverenti che influenzassero in modo determinante la morale collettiva, fino a mancare di rispetto a un sacramento quale il matrimonio. ${ }^{7}$ Nei paesi cattolici dell'Europa meridionale, le scampanate assumono una maggiore clandestinità e divengono poco praticate dai ceti superiori, mantenendo tuttavia la propria vivacità in ambienti popolari, soprattutto rurali; ma tanto in Italia come in Spagna tale inibizione ha effetti sulla letteratura, e l'argomento viene rimosso, o per lo meno mascherato. Nell'avviare la moda dei poemi eroicomici, nel 1618 Francesco Bracciolini - poeta al servizio del futuro papa Urbano VIII - nel suo Lo scherno degli dei non fa il minimo cenno a simili forme di satira, per quanto l'argomento si presterebbe notevolmente, dato che deride i costumi sessuali dell'Olimpo secondo la mitologia greca. Pure nella Fiera di Michelangelo Buonarroti il Giovane, pubblicata pochi mesi dopo, si fa appena un minimo cenno alla derisione riservata a una prigioniera 'trascinata a suon di corni il dì di Befania'. 8

Solo in due opere in prosa, antispagnole e polemiche verso il papato, si arriva a parlare liberamente di scampanate, per di più fatte per satira politica: i Ragguagli di Parnaso di Traiano Boccalini, e La baccinata ovvero battarella (due termini per designare la scampanata, rispettivamente a Roma e a Venezia) di Ferrante Pallavicino. All'inizio del XVII secolo, i due autori, pur in forma anonima, possono pubblicare simili opere solo nella Repubblica di Venezia, all'epoca in urto con Roma. Entrambe le opere, subito percepite come eretiche, vengono immancabilmente inserite nell'indice dei libri proibiti e - circolando clandestinamente, o tradotte nei paesi protestanti - divengono presto dei classici dell'antipapismo. La baccinata, in particolare, porterà Pallavicino sul patibolo di Avignon, appena ventottenne. Visti questi precedenti, che mettono in allarme sia gli scrittori libertini che quelli più ortodossi, in Italia nessuno scriverà più di baccinate o battarelle fino al XIX 
secolo. Successivamente, un fugace accenno al fracasso d'una scampanata dal millantato scopo esorcistico c'è nella favola Belfagor l'arcidiavolo, messa in terzine all'inizio del XVIII secolo, rielaborando il celebre testo di Nicolò Machiavelli, dal poeta bernesco fiorentino Giovan Battista Fagiuoli: 'Abbian trombe, tamburi, e cornamuse, / Cemboli, corni, pentolaccie, e cose, / Che a far romor da diavoli sian use. / [...] Vengan via con sonate strepitose'. ${ }^{9}$ Rime dichiaratamente ispirate a uno degli scritti rinascimentali più contrastati dalla censura ecclesiastica, perciò subito poste all'indice dal Sant'Uffizio.

\section{Ultime fischiate ai cavalieri}

La tradizione italiana dei poemi eroicomici ha tra gli ultimi strascichi il Ricciardetto di Niccolò Forteguerri, pubblicato a Venezia nel 1738, tre anni dopo la morte dell'autore, un letterato pistoiese di famiglia nobile, abate, segretario di papa Clemente XII. ${ }^{10}$ Riproponendo fuori dal suo tempo il poema eroicomico, l'autore rende omaggio a un mondo in lento declino, dove la festa di piazza può ancora portare la sociabilità e i modi di esprimersi dei diversi ceti sociali a incontrarsi coralmente, anche in rima:

lasciata la pompa e il decoro, le donne illustri e i cavalieri eletti disceser nelle piazze, e tra coloro di bassa riga allegri si meschiaro e con essi lietissimi ballaro.

Là sentivi cantare all'improvviso uno straccione a rispondergli un oste, $[\ldots]$ e vicine e discoste le genti applaudire a piene bocche. loro versi strani e lor rime sciocche. (Niccolò Forteguerri, Ricciardetto, XXX, 17-18) ${ }^{11}$

Un variegato coro di spettatori - che ridono o si commuovono dei casi bizzarri, inquietanti, grotteschi o cruenti in cui incorrono i personaggi - è regolarmente presente in quest'opera, soprattutto nel canto XXX, quello conclusivo. D'altronde, è costume degli autori dell'epica eroicomica 'accomodarsi all'idee e opinioni della moltitudine', secondo quanto deplora nel 1772 il principale censore italiano delle scampanate, il sacerdote Bartolomeo Napoli. ${ }^{12}$ Intende così mettere in rilievo il suo biasimo al loro modo di narrare diversi passaggi avventurosi delle vicende guerresche con una trattazione di ambientazione e linguaggio basso-plebei. Va notato, però, che tutto il poema, com'è costume per gli autori colti fino alla fine del XVIII secolo, non si preoccupa di descrivere la vita popolare, facendo a ciò appena qual- 
che accenno occasionale. Nel poema, così, anche le sonore derisioni ad alcuni eroi non sono attribuite a genti europee, ma a diavoli o strani popoli di isole lontane, con solo vaghe sembianze umanoidi.

Queste derisioni sonore sono tuttavia molto ricorrenti. Tanto più che ad alleggerire con una nota comica la prolissa narrazione non sono tanto i giganti derivati da Boiardo o da Rabelais, che qui appaiono solo come guerrieri terribili e, nel raro caso di don Tempesta e don Fracassa, anche convertibili alla vita cristiana come pii sacerdoti. Sono invece principalmente due personaggi - i cavalieri Ferraguto (più spesso abbreviato in Ferraù) e Astolfo - che si trovano ripetutamente nella condizione di perdere l'onore e subire scampanate, per posizioni scabrose in cui di continuo cadono, incapaci di controllare i propri impulsi sessuali. La mancanza di autocontrollo è attribuita dall'autore a molti nobili cavalieri, celebrati per il loro temibile valore guerriero. Ma gli altri cavalieri cercano di affermarsi solo per la forza smodata del loro braccio, o - eccettuati i frequenti ingannevoli incanti che si frappongono per rendere intrigante all'estremo la storia - s'innamorano perdutamente di una sola donna; mentre questi due si caratterizzano come subdoli seduttori, pronti a inseguire ogni possibile situazione di appagamento sessuale - Ferraguto persino con un orripilante mostro diabolico - sempre finendo in posizioni grottesche, contrastanti col loro riconosciuto valore di terribili soldati.

È poi interessante vedere come in questo poema i personaggi risultino fortemente sensibili alle dicerie sul proprio operato, soprattutto se si tratta di imbarazzanti racconti veritieri, lesivi dell'orgoglio virile o della loro immagine di cavalieri protettori dell'onore di fanciulle. I due campioni citati incorrono di frequente in simili situazioni sconvenienti. Ferraù si sente spesso nella condizione 'onde da ognuno sarà villaneggiato' ( $R$ VII, 63); supplica perciò i testimoni delle sue gesta poco onorevoli di mantenere il silenzio e conta sul fatto che l'isola dove ha commesso le sue peggiori manchevolezze e dove ha subito una clamorosa scampanata 'è si lunge / che fama pur di lei qui a noi non giunge'. Perciò alla corte di Carlo Magno si mantiene reticente su 'come amor piagollo e vinse / per un demon, per una furia espressa' ( $R$ XIII, 69, 70). Astolfo, a sua volta, preferisce evitare risse dopo aver dato scandalo, 'ché non vorrebbe andar su le gazzette' ( $R$ XVIII, 58). Tuttavia i loro tentativi di tener celate le proprie peripezie disonoranti, soprattutto erotiche, riescono immancabilmente vani. Le derisioni, così, incombono su questi personaggi di condizione nobile, o sul clero stesso, non tanto su gente del popolo. A ridere di questi eroi, che loro malgrado finiscono per essere percepiti come buffoni, sono a varie riprese la corte di re Carlo, i diavoli dell'inferno e il popolo del contado; uomini e donne di chiesa appaiono gli unici a non riderne mai. Paladini e nobili abbinano invece alla derisione il compatimento o il biasimo per qualcuno che scredita il proprio ambiente cavalleresco.

Ferraù è un valoroso cavaliere musulmano convertitosi al cristianesimo, che si fa penitente frate eremita. Quando Carlo Magno chiama i suoi paladini a 
difendere Parigi dall'invasione di imponenti eserciti saraceni venuti dall'Africa, riprende le armi e accorre a combattere a difesa della Francia e della Spagna cristiane. La sua incontinenza sessuale, più ancora di quella del cavaliere inglese Astolfo, è però origine di molte vicende comiche, da cui monsignor Forteguerri trae occasione per lunghe tirate volte a sollecitare la chiusura dei conventi maschili, allo scopo di arginare l'ipocrisia di un clero regolare che vede soggetto a continue sregolatezze alimentari e scandali sessuali, dietro la facciata del saio ascetico francescano. La comicità di questo frate guerriero viene dalla sua focosa passionalità, che lo porta d'impeto a proclamare la più decisa volontà di mortificazione della carne, mentre si invaghisce fulmineamente di qualsiasi donna - o anche solo essere femminile - che incontri.

Per tutti i personaggi, le più cocenti derisioni non avvengono nella quotidianità consueta dei campi di battaglia, ma peregrinando in varie isole lontane dall'Europa. Tali isole non appaiono però tanto luoghi di dannazione, quanto di incanti bizzarri o di meraviglie esotiche quasi naturali: per i personaggi più puri potrebbero anche convertirsi in un percorso di prove iniziatiche, dato che i pericoli che si prospettano non risultano reali e le aggressioni appaiono solo simboliche. In particolare nell'isola del Portento, 'sfortunata e ria isola, fatta di folletti sede' ( $R$ XIII, 5), 'isola de' scherzi e del travaglio' ( $R$ XIII, 7). Può apparire un paese di prodigi carnevaleschi, un incantato mondo alla rovescia, dove la necessità delle burle stupefacenti rende sempre le cose diverse da quel che sembrano, a causa dei 'dispetti [...] da quei diavolini / che fanno i buffoncelli e i mattaccini' ( $R$ XII, 107).

Quest'isola per voce antica molto

è fama che alberghino i folletti, che fan con tanti scherzi ogni uomo stolto:

or tiran le lenzuola di su i letti, or prendon di donna o d'uomo il volto, or si fanno orsi, or gatti, or micetti, in somma chi s'abbatte in questo loco diviene di color favola e gioco.

Ma non fan male alcuno, anzi sovente fanno del bene e insegnano tesori e modi di campare allegramente e di birbanti divenir signori. ( $R$, XII, 83, 84)

Transitarci può essere dunque un modo per emendarsi dalle sciocchezze, imparare a vivere piacevolmente e con ironia, e fortificarsi nel carattere, avviandosi a una nuova vita. E numerosi eroi vi subiscono gli scherzi più meravigliosi. Appunto perché, secondo Forteguerri, scampanate e beffe potrebbero diventare strumento di redenzione, per gli stessi eroi, come accade ai figli ventenni di Orlando e Rinaldo. Non così per Ferraù e Astolfo - a ben guardare - che nelle 
diverse isole incorrono nelle umiliazioni e negli scandali più estremi, ritrovandosi fragili e in uno stato di prostrazione e isolamento da cui non usciranno indenni nell'onore e neppure fisicamente, sebbene sia il frate-cavaliere a liberare i giovanissimi eroi, e anche i loro padri, Orlando e Rinaldo, e tutti gli altri cavalieri da quel labirinto di burle.

Ferraù vi giunge in veste di missionario, coadiuvato dai suoi monaci giganti, e fanno ridere cercando con piviale e aspersorio di gridare esorcismi e preghiere per scacciarvi i diavoli, mentre i diavoli si prendon gioco di loro, facendo anche piovere loro addosso sassi e un immaginario grande serpente. Ma il missionario ritrova lì l'eroina musulmana Climene, che gli ha già in precedenza fatto perdere ogni ritegno, involontariamente riducendolo con la propria bellezza a 'quel gran cornuto / che di lussuria portato dall'estro / fece di Cristo il perfido rifiuto’ ( $R$ VII, 60). Perciò, lei col paladino Guidone che ama, e il paladino Ricciardetto con la sua amata, 'dieder'n un riso sì spropositato' ( $R$ XIII, 35$)$ nel vedere il frate - fino a poco prima arso dalla libidine - lanciare scongiuri contro i diavoli, vestito cerimonialmente con cotta e piviale. Allora lui scambia i nuovi arrivati per una malefica apparizione soprannaturale e coi compagni giganti cerca prima di farli scomparire con inutili esorcismi, poi di impugnare la spada e combatterli con furia, finché lei riesce a palesarsi per quel che materialmente è, convincendolo - senza tante cerimonie e con palese allusione oscena - appena 'piglia in mano il suo cordone / e al romito vien la tentazione. // E lo leva sì tosto di cervello / che l'asperges gli cade giù di mano' ( $R$ XIII, 41-42). Lui a quel punto, eccitatissimo, vorrebbe buttare immediatamente alle ortiche la propria castità, con esibizionismo esuberante, pur vedendo Climene assieme al paladino suo fidanzato. Ma il gigante don Tempesta, arrossito di vergogna, allora cessa di recitare scongiuri dal proprio breviario e - passato a misure pragmatiche-imprigiona il frate nella propria rete indistruttibile, mentre i paladini e le loro dame musulmane non riescono a dissimulare le proprie emozioni, come vorrebbero fare ('Climene e gli altri ne fanno gran festa, / e la furbetta con sembianze liete / gli va d'attorno e, vistolo in tal guisa, / pianger vorrebbe e le scappan le risa'; $R$ XIII, 52). Non serve altro: subito si scatena a deridere il missionario una sarabanda di diavoli che fanno una vera e propria scampanata, che l'eclettico e sbrigativo Forteguerri descrive però in veste di spettacolare carnevale.

E quindi risonar l'isola tutta

s'ode di pentolaccie e di fischiate:

come di carneval quando in bautta

ed in maschera vanno le brigate

che in larga piazza la gente ridutta

in vedendole falle le risate,

così i demoni a vederlo in quel modo

ridevan fra di loro sodo sodo. ( $R$ XIII, 53) 
La scampanata però viene fatta cessare da un ennesimo esorcismo del missionariogigante don Tempesta, che libera definitivamente l'isola dalle orde di diavoli. Solo allora Ferraù si copre di vergogna e lacrime, prima che il rapimento di una delle dame trascini tutti di nuovo in mare, facendone finire alcuni in 'un'isoletta bella ed aprica', ma abitata da un 'popolo brutto molto e scontraffatto assai' ( $R$ XIV, 44). Il 'popolo strano' $(R \mathrm{XIV}, 55)$ che la abita, gente nuda, senza alcun pelo e sporca, con pelle verde e mani lunghissime, trova invece bruttissimi i paladini e gli esseri umani coi loro capelli e peli, perciò 'incominciaro a far risa da matti / parendo lor che fossero orsacchini / o simili animali scontraffatti' ( $R$ XIV, 47). Orlandino e Nandino - simili a Cortez o Pizarro nelle Americhe - non sopportano 'quello così pazzo riso' di 'questi burattini, queste scimmie, questi brutti gatti' ( $R \mathrm{XIV}, 48,47)$, così ne fanno strage, con esuberanza incontrollata.

Sopravvivono il re e la regina dell'isola, che si umiliano e pacificamente rendono onore coi superstiti ai giovani paladini nuovi venuti, con grandi feste a palazzo. Ma gli ospiti riprendono a farvi strage per capriccio, così che quando contro Orlandino si scaglia un mostro nume tutelare del luogo, come segno di gioia e approvazione - nell'erronea convinzione che il figlio di Orlando sia stato sprofondato nel terreno e ucciso - 'l'isola ne fea un gaudio strano / con corni e pive e battere di mano' ( $R$ XIV, 78), prima di ricevere ulteriori tormenti e sopraffazioni dagli altri paladini giunti nel frattempo, coi monaci-giganti. Questi ultimi e Ferraù tolgono dai guai i due giovanissimi paladini e le loro fidanzate, poi il frate vi rimane a predicare, per convertire al cristianesimo tutta la popolazione, contando che la bruttezza delle donne locali non lo indurrà in tentazione. Alla prova, però, entro un mese s'invaghisce della più bestiale 'cosa' abitante dell'isola, 'strega maledetta' e 'mostro della terra' ( $R$ XV, 65, 68); salpa con lei verso la Francia, tra la costernazione dei suoi compagni giganti don Tempesta e don Fracassa, mentre i marinai che li accolgono su una nave, ridendone ('stimaro il frate de' pazzi il monarca'; $R \mathrm{XV}, 68$ ), e allora l'innamorato forestiero li passa tutti a fil di spada, per punirli di una pur non clamorosa derisione. Tutte le creature viventi e quelle soprannaturali del mare - da Nettuno a Scilla e Cariddi-accorrono a sbellicarsi dalle risate, vedendo navigare questa coppia insensata, finché interviene la dea Teti a trasformare lei in una seppia, per farla sembrare meno brutta, e poi la separa dal frate.

\section{La provincia cerca i propri Ossian}

Col diffondersi dell'interesse folkloristico, anche in luoghi molto periferici si riscoprono ed estetizzano le culture agro-pastorali e le parlate dialettali. Se ne trova un caso nell'entroterra di aspre montagne della Repubblica di Genova, vicino al crinale delle Alpi marittime e al confine col Ducato di Savoia e con la contea di 
Nizza. Nella seconda metà del XVIII secolo, l'avvocato Luca Maria Capponi, nei casolari di Gerbonte, attorno al villaggio di Triora, scrive in rime dialettali $A$ canzún der pegurò, la canzone del pecoraio Franzé (nome leggibile come Francesco, ma anche come il francese). Difficile dire se questo scritto volesse inizialmente avere un utilizzo per letture ad alta voce in ambito domestico, oppure in circoli della ristretta élite dei borghi montanari. Il poema rimane inedito e probabilmente viene divulgato in forma orale, o ricopiandone i versi. Stando alla testimonianza di un monaco romano originario di Triora, che a metà del XX secolo ne pubblica i primi tre canti, 'copie manoscritte di questa composizione circolano da oltre un secolo in Triora e vengono lette con viva curiosità. Vi sono persone che ne recitano dei brani a memoria con la stessa facilità del Pater noster.${ }^{13}$ Dalla gente del paese questo racconto viene definito fôra, una favola. Il manoscritto è andato perduto nel 1944, quando truppe tedesche hanno bruciato parte del paese per rappresaglia. Degli altri sette canti restano solo i titoli, in parte trattanti le difficoltà della vita matrimoniale e del cercare moglie da vecchi. Si tratta sempre di riflessioni senili attribuite da Capponi al rude e povero pastore e casaro settantenne Franzé, vedovo ormai privo di forze e appena fugacemente animato da passioni: un tempo rispettato per il suo vigore e le sue bravate paesane, ma da vecchio ridotto quasi a una macchietta pittoresca.

Il rituale descritto nel secondo canto del poema - riprodotto qui nell'Appendice I - descrive una forma particolare di scampanata, i ciaravügli del Ponente ligure e delle Alpi marittime, la cui dizione si avvicina al piemontese ciabra e ancora più al francese charivari. ${ }^{14}$ In questo caso, il deriso è l'uomo vedovo che sposa una vedova, e pare avere come consueto la possibilità di scegliere se arrivare subito a una transazione coi giovani del paese, offrendo loro vino e frutta, oppure continuare a subire la derisione fino al cedimento di una delle due parti. Accettando subito di trattare con la banda rumorosa, che mostra proprie gerarchie interne e una discreta autodisciplina, però, il deriso diventerebbe il rettore riconosciuto di quella specie di badia che va a deridere i vedovi quando si risposano. In un'alternanza ciclica, il deriso erediterebbe dunque il ruolo carnevalesco di organizzatore della successiva scampanata, venendo a tale scopo dotato di abito e cavalcatura ridicoli. Se rifiutasse di trattare, con la forza sarebbe invece caricato a cavalcare alla rovescia su un asino sofferente alla schiena, che farebbe perciò di tutto per disarcionarlo. Il fatto che il racconto prenda in considerazione lo svolgimento del rituale meno aggressivo e più benevolo possibile, mostra un chiaro interesse dell'autore a non presentare in modo inquietante la tradizione locale, a mostrarla come un collante normale e necessario delle relazioni paesane. Questo avvocato montanaro pare condividere le usanze locali, pur inclinando a considerarle curiosità ormai arcaiche di un mondo che sta declinando: se non fosse per un certo distacco paternalistico che traspare nel suo linguaggio verso i ragazzi scatenati e la figura patetica del 
pastore, si potrebbe dire che vede semplicemente in questo rituale un'animata festa carnevalesca e un rito di passaggio, momento vitale della sociabilità paesana.

L'aggregazione della gioventù celibe come un vero e proprio corpo sociale informalmente costituito resta a lungo a presiedere alla vita paesana e di microcomunità isolate, più che non a quella cittadina. Una delle sue fondamentali funzioni è di vigilare sui comportamenti collettivi. Il paese riconosce a questi gruppi informali dei diritti di questua, che corrispondono a un ruolo che tali aggregazioni hanno nel regolamentare determinati meccanismi della convivenza paesana, in particolare le scelte matrimoniali, che così non sono dettate unicamente da alleanze tra gruppi di famiglie, ma sono oggetto di mediazioni più complesse, in cui anche i giovani assumono qualche voce.

Il ciaravüglio viene visto da Capponi come dotato di un proprio ordine interno, non come l'esibizione caotica descritta invece da molti altri autori italiani del secolo successivo. ${ }^{15}$ Questo autore insiste nel mostrare come i responsabili di simili rituali si preoccupino di vedersi riconosciuti scopi morali nel proprio agire, e un retto operare nel far valere il rispetto della tradizione, anche se a legittimarli non appaiono in alcun modo simboli e atteggiamenti rinviabili alla religione. Simboli religiosi delle badie sono ancora riscontrabili alla fine del XVI secolo (ad esempio nel Gioco d'Acheronte di Alessandria, come si vedrà), mentre paiono sempre meno presenti nelle residue aggregazioni dei celibi dopo la metà del XVIII secolo, in un contesto di accentuata secolarizzazione. Piuttosto che una corrispondenza col priore di una confraternita religiosa, il capitano appare - nel costume rituale, oltre che nella sua qualifica, pure in forma carnevalizzata - un ruolo che, in determinati momenti, potrebbe assumere funzioni di direzione paramilitare della gioventù nel borgo montanaro. Un ruolo sostanzialmente in linea con la prassi di bravate dove i giovani esibiscono armi o bastoni, assunzione di una direzione profana nella gestione di feste locali, azioni collettive galanti, occasioni frequenti per danzare fuori dal consueto tempo del carnevale, ma anche momenti di turbolenza municipale, che questo genere di associazioni si assume, nella stessa epoca, anche nell'attigua regione provenzale. ${ }^{16} \mathrm{E}$ - almeno nel racconto di Capponi - nessuna autorità civile o ecclesiastica appare mentre si fa rumore: né per legittimare il rituale popolare, né per contrastarlo. Simili aggregazioni municipali dei giovani, come le figure di questi capitani, inevitabilmente si vanno sempre più folklorizzando, mentre gli stati iniziano a strutturarsi con crescente efficienza nell'organizzare propri apparati militari centralizzati, che rendono superflua la mobilitazione di milizie campagnole guidate dalla nobiltà locale.

Nelle città del XVII e XVIII secolo, intanto, le scampanate sono considerate dalle autorità civili e religiose, come dai ceti borghesi, una grossolana giustizia informale, cui non viene più riconosciuta alcuna ufficialità, ma che - per non costringere a continui impopolari interventi repressivi le forze di polizia - può continuare a essere tollerata, se si mantiene nella marginalità, senza provocare 
incidenti. A Torino, dove le autorità cittadine hanno vietato con severità la chiabra dei mariti fin dal 1343, neppure il susseguirsi di divieti e punizioni decretate anche dalle autorità diocesane - in sei sinodi dal 1500 al 1670 - ottiene di far scomparire l'usanza, pur limitandola nelle sue intemperanze originarie; tra XVI e XVII secolo situazioni analoghe si riscontrano nelle vicine diocesi di Vercelli, Alba e Ivrea. ${ }^{17}$ A cavallo tra XVIII e XIX secolo, ciò pone però problemi nuovi, che assumono un peso rilevante nella contrastata transizione dall'ancien régime alla civiltà borghese. Nel momento in cui sono dei leader popolari a guidare le usanze dei giovani formalmente messe all'indice e fuori dalla legalità, questi hanno meno possibilità di mediare in precedenza un pegno da far pagare per non subire la derisione notturna che, per le sue sonorità e i suoi cortei (sempre più infrequenti nelle città), continua ad assumere un carattere pubblico, mentre formalmente si tratta di un rituale clandestino. Dovendo mantenere clandestina la scampanata, inevitabilmente i partecipanti si concentreranno sul tenere segreta la sua attuazione fino all'ultimo momento, piuttosto che tentare patteggiamenti preventivi, di cui è facile prevedere l'insuccesso, data la possibilità di incorrere in denunce per tentativo di estorsione.

Nonostante ciò, dalla seconda metà del XVIII secolo, poi ancora nel corso del XIX, si nota in diversi paesi europei un'espansione delle pratiche delle scampanate e delle loro funzioni derisorie, estese a molti altri aspetti della vita familiare e sessuale, ma anche - e con crescente insistenza - a particolari comportamenti sociali, economici e politici malvisti dalla comunità locale, per sottolineare l'isolamento scandaloso del soggetto che ne sia indicato responsabile. Parallelamente, a contrastare questa tendenza, la pratica di queste derisioni notturne dall'inizio del XIX secolo incontra crescenti opposizioni da parte delle persone derise, sia perché vedono oltrepassati i codici consuetudinari e si vedono vessate dai compaesani per ragioni anomale, sia perché la mentalità borghese ora ritiene volgari, offensivi, violenti e privi di senso simili rituali comici. Autorità e forze dell'ordine si mostrano maggiormente sensibili a tali sollecitazioni e denunce, vedendo in queste pratiche una sospetta tendenza alla trasgressione delle leggi e a deridere possibili sostenitori dell'ordine costituito, col pretestuoso paravento del rispetto a usanze tradizionali della gioventù del luogo. ${ }^{18}$

\section{Un tardivo epos della passata crociata anticarnevalesca nelle città}

Il Ricciardetto visto poc'anzi è un'opera per la lettura ad alta voce per l'intrattenimento nelle accademie arcadiche e nei salotti aristocratici, più che destinata alla stampa. Solo dopo la morte del suo autore il poema viene stampato e ha una discreta fortuna, per quasi due secoli. Ad Alessandria, nel 1834, un poemetto manoscritto (Il Gioco d'Acheronte) con simili funzioni d'intrattenimento 
accademico e salottiero, ma con un soggetto che abbandona la narrazione di avventure fantasiose dei paladini, per orientarsi invece alla riflessione storicofolkloristica appropriata alla cultura romantica, è opera di Pietro Gaspare Melazzi (1789-1842), conte di Valle S. Bartolomeo, membro e animatore dell'Accademia poetica degli Immobili.

Nel periodo della Restaurazione, Melazzi è stato un personaggio pieno di iniziativa, con un protagonismo debordante e munifico nella vita culturale di Alessandria. Erudito cultore d'arte e letteratura, con una morbosa passione per la storia cittadina, le gambe paralizzate da una malattia, passa la vita a scrivere un'eccezionale quantità di composizioni in versi, di mediocre qualità dilettantesca, rimaste prevalentemente inedite. Melazzi compila anche un poema per celebrare la messa al bando nella sua città di una forma di derisione carnevalesca alle vedove risposate; ${ }^{19}$ derisione che fino al XVI secolo aveva luogo di giorno, senza che nei documenti siano nominati baccani fragorosi fatti con occasionali strumenti da percussione. Le informazioni storiche le trae direttamente da una cronaca cittadina stampata nel 1666. Sia quella cronaca, sia altre precedenti cospicue opere poetiche scritte tra XVI e XVII secolo, e ancora un'altra storia locale scritta a due secoli di distanza (poco dopo la morte di Melazzi) condividono l'entusiasmo di questo nobile per l'opera sistematica svolta dal clero locale dopo il concilio di Trento, per contenere e scomunicare le esuberanze della cultura carnevalesca, praticate sia dall'élite che dal popolo. ${ }^{20}$

Il nobile alessandrino rielabora in uno scorrevole linguaggio in versi, per renderle comprensibili nella sua epoca, le notizie che ritrova nella cronaca manoscritta di quasi due secoli prima, opera dell'annalista municipale Gerolamo Ghilini. In quella cronaca si elogiano il vescovo Guarnero Trotti e le autorità locali, per avere dal 1584 proibito una tradizione fonte di disordini e ricorrenti violenze: il gioco d'Acheronte, scampanata diurna fatta da giovani mascherati. Per celebrare la difficile e innovatrice opera censoria di queste autorità, l'annalista ripercorre con un'ampia trattazione quali fossero gli eccessi delle locali scampanate e pratiche carnevalesche. La cronaca di Ghilini era ben sintonizzata col clima della Controriforma, che ad Alessandria aveva già prodotto, in ambienti accademicoecclesiastici, un poema dove - trasponendo in versi poetici la filosofia morale ispirata ai dettami tridentini - si fustigavano le prepotenze e le volgarità oscene del carnevale. ${ }^{21}$

Tale proibizione, avvenuta due secoli e mezzo prima, Melazzi la presenta come vittoria della morale cristiana e civile sui barbari residui del medioevo nei costumi municipali. Una visione che apparirebbe di segno illuministico, se la morale epicizzata non fosse quella di un nuovo ordine autoritario alla De Maistre, che possa spianare anche le tradizioni civiche, pur di imporre un ferreo rispetto del clero disciplinatore: una morale dunque consona al legittimismo reazionario della Restaurazione, che solo nel recupero del potere assoluto e del disciplinamento 
religioso vorrebbe ritornare ai costumi del XVIII secolo, ma che non intende ripristinare il vigore dei costumi popolari sia urbani che rurali, al di là di certe forme esteriori, estetizzate dalle mode romantiche. Qualunque dimostrazione tumultuosa viene ora vista con spavento dai tutori dell'ordine e dai maggiorenti, che dietro ogni disordine - anche quello ritualizzato dalla tradizione carnevalesca - intravedono possibile lo spuntare di alberi della libertà, barricate e ghigliottine.

Il conte Melazzi ha così modo di fare dell'archeologia del costume cittadino, rivisitando una cronaca manoscritta vecchia di un secolo e mezzo, ma immettendo nella sua riscrittura in versi abbondanti elementi fantastici, assieme a valori politici e inquietudini della propria epoca, nel più autoritario degli stati italiani: il Regno di Sardegna. Riflessioni che possono dar bene la misura del clima in cui - sempre in una provincia sabauda - prende un significato sociale e politico trasgressivo la scampanata, descritta in seguito in un romanzo inglese di Giovanni Ruffini, ambientata però, e forse davvero vissuta, dall'autore negli stessi anni in cui Melazzi scrive le sue sestine sul gioco d'Acheronte. ${ }^{22}$ Trattando di queste derisioni, la scelta di Melazzi è del tutto antitetica a quella di Ruffini, che da giovane era aiutante del leader rivoluzionario Mazzini. Le derisioni alle vedove lo interessano solamente come un curioso costume pittoresco di un lontano passato; non spende nemmeno una parola per accennare alla permanenza di rituali chiassosi notturni molto simili, rimasti comunemente praticati tanto nella sua città come nei suoi possedimenti nel contado tra il Tanaro e il Po.

Trattando della figura delle vedove, nel poema emerge pure una tendenza della civiltà romantica: dato che la giovane Isabella è presentata realmente ardente d'amore, in nome di tale sentimento viene giustificata come ovvia la sua possibilità di fare allora scelte che per Melazzi non appaiono più controcorrente. E la chiesa cattolica che nel XVI secolo continuava a biasimare le seconde nozze come una forma appena tollerata di bigamia, viene invece da lui presentata come organizzatrice di una cerimonia nuziale sfarzosa e con il clero compattamente schierato a difesa del rito matrimoniale della vedova, contro ogni interferenza profana e ogni possibile satira denigratrice. Ma la condiscendenza di Melazzi verso la giovane vedova non va oltre il poter ammettere un secondo matrimonio: non si arriva a concepire alcuna reale autonomia della donna, e Isabella decide le nozze solo dopo aver avuto il pieno consenso dei propri familiari, passando da quel momento sotto una possente tutela della famiglia dello sposo.

Se nel poemetto è attratto da un interesse antiquario per un cimelio cittadino delle badie dei giovani aristocratici (un gonfalone carnevalesco con l'insegna della capra), Melazzi non mostra nessun interesse a riconoscere una qualunque logica all'agire di questa gioventù, coi suoi metodi violenti sopraffattori. Questa viene senza esitazione presentata come ispirata dalle peggiori forze del male, anche se molti dei componenti della badia carnevalesca sono identificati come appartenenti a famiglie cittadine di spicco. Come forze ideali del bene sono invece rappresentate le 
schiere del clero guidate dal vescovo Trotti, che, in ottemperanza alle deliberazioni del concilio tridentino, combatteva con veemenza molti usi profani carnevaleschi. Monsignor Trotti imponeva che tali restrizioni cominciassero proprio dalla messa al bando dell'invadenza violenta e irridente delle badie giovanili - presentate da Melazzi come 'la perfida genì' ( $G$ III, 20) da combattere del pari dei turchi appena battuti a Lepanto - nel rituale satirico dell'Acheronte, riuscendo ad avere anche le autorità municipali dalla propria parte. Alle spalle del vescovo punitore della ritualità profana, emerge come eroina la vedova vittoriosamente ribelle alla brutalità di 'questa [...] usanza che funesto ha scopo' (G III, 15), che macchia irrimediabilmente l'onore delle donne derise. L'autore non manca poi di introdurre distinzioni nette tra le generazioni più giovani: tra la compagnia di 'quei che di virtù seguon la via' ( G II, 36), e un'altra dedita a trivialità goliardiche.

L'influenza culturale della Restaurazione è evidente subito dai primi versi dell'invocazione iniziale, dove le stesse muse del Parnaso, qui protettrici del tardo classicismo e probabilmente dell'Arcadia, divengono suore cacciate 'nel più cupo orrore' ( $G$ I, 1) da un luogo sacro distrutto. Un'immagine di rovine di conventi in cui non è difficile scorgere l'evocazione di un lungo strascico tempestoso della rivoluzione francese, di quattro decenni precedente, secondo una tendenza ben presente nel nuovo gusto romantico del suo tempo: moda artistica con cui l'autore stesso ammette di doversi contaminare, pur in parte biasimandola. ${ }^{23}$ Si dilunga poi a spiegare come i consigli di amici più indirizzati verso i classici gli siano preziosi ('onde le traccie seguirò degli Avi', G I, 7): non solo gli antichi, ma Dante, Petrarca, Tasso, Ariosto, e soprattutto 'Tu, Berni, che mi fai sì lieta / L'alma ogni volta che ti leggo, sia / Propizio a chi vorrebbe esser Poeta / Ma aver la natural tua leggiadria' ( $G$ I, 9). Omaggio sorprendente, quello a Berni, visto che di comico e di satira nei versi di Melazzi non c'è neppure l'ombra. Anche nel descrivere i disordini provocati dalla 'scapestrata gioventù' ( $G$ IV , 7), l'argomento è piuttosto inframmezzato da innumerevoli divagazioni, anche molto lunghe, sulla storia municipale, o su allegorie mitologiche, o ancora con estemporanei commenti sui costumi alla moda.

L'epoca evocata nel poema è la fine del XVI secolo, ma l'immagine che se ne rende rassomiglia moltissimo a quella tutt'altro che idilliaca del XVIII secolo appena conclusosi coi suoi rivolgimenti politico-sociali, coi difetti che la cultura della Restaurazione imputa alla decadenza dell'aristocrazia che non ha saputo conservare l'ancien régime:

Erasi il tempo, in cui vicino al foco

Da tutti si trapassavano le sere,

Ovver danzando, o delle carte al gioco

Fonte di pianto a molti, e di piacere

A pochi a pochi assai, perché da questo

Nascer ne suol effetto assai funesto. ( $G$ I, 10) 
La vicenda prende avvio da una sciagura avvenuta nel Carnevale 1577 ad Alessandria, in occasione della solenne serata danzante in un palazzo nobiliare dov'erano convenute molte famiglie dell'élite locale, assieme a nobili spagnoli. Ceduto il pavimento di un salone dove erano assembrati gli uomini, molti morirono o rimasero seriamente feriti, mentre la maggior parte delle donne restò incolume. Per un anno fu stabilito il lutto cittadino, con sospensione di tutte le feste. Tra le vittime, Muzio Bandolino, i cui averi passarono alla giovane vedova Isabella dei Marcelli, che osservò inizialmente un lutto stretto, curata dai vicini a cui la sua famiglia la raccomandava, in particolare da un giovane suo amico d'infanzia, Zampiero Cellerino. I due finirono per stabilire segretamente di andare a nozze, una volta trascorso abbondantemente l'anno di lutto. Nella realtà storica, l'azione del vescovo di proibire le derisioni per le seconde nozze è di diversi anni successiva, e conseguente non a un episodio locale, ma a un generale orientamento della chiesa cattolica, sollecitato dal cardinale Carlo Borromeo. Nel poema, invece, i due diversi episodi vengono fatti coincidere, per trovare una storia esemplare che giustifichi le restrizioni clericali.

All'inizio del XIX secolo, nel clima tetro e bigotto della Restaurazione sabauda, Melazzi non si sente minimamente portato a prendere le difese dei vecchi costumi carnevaleschi, né delle scampanate, che pure, in tono minore, si continuano a fare nei rioni cittadini e nelle campagne circostanti. Vede semmai l'Acheronte come una curiosità storica, da rendere oggetto di divulgazione colta, consapevole di offrire così un argomento gradito ai gusti antiquari dei circoli accademici cittadini dell'epoca romantica, mostrando un costume dei loro antenati alla stregua di uno stravagante rozzo esotismo. D'altronde, in Italia, proprio in Piemonte - come nella contigua Francia - le badie giovanili che gestivano tali rituali hanno avuto un particolare rigoglio, e saranno a lungo oggetto di un interesse di carattere storico per gli studi di filologi, giuristi e folkloristi, appassionati rivisitatori delle antiche forme associative giovanili, in particolare di quelle dei ceti aristocratici e istruiti. ${ }^{24}$

Nella cultura neoclassica e romantica, la riscoperta di rituali da indirizzare pedagogicamente al popolo non è una rivalutazione indiscriminata delle tradizioni, né in campo civile né in quello religioso. In modo deciso, invece, nella lunga transizione tra il XVIII e il XIX secolo - tra lo spegnersi dell'ancien régime e l'affermarsi della civiltà borghese - le feste sono proposte come esemplificazioni di una nuova razionalità civile e religiosa, sia quando a essere evocata è la razionalità dell'età dei Lumi, o della Rivoluzione, o invece della Restaurazione. Nelle ritualità rivalutate non può esserci posto per quelle con caratteristiche grossolane e violentemente sguaiate, come le tradizioni carnevalesche, o - in ambito religioso - come le raccapriccianti processioni di flagellanti rievocanti la passione di Cristo, o molti pellegrinaggi, o anche processioni notturne, che davano abitualmente occasione a disordini e intemperanze, nel XIX secolo mal tollerate. ${ }^{25} \mathrm{Al}$ di là della constatazione che in Alessandria i gruppi che fino al XVI secolo si riconoscevano 
nello stendardo dell'Acheronte si sono veramente estinti, quello avvertito in modo forse inconsapevole da Melazzi è un problema ormai secolare che si ripropone ancora nella prima metà del XIX secolo: non pare più il tempo per ritualizzare il disordine, né per dare spazio alla satira, e neppure per mettere a stretto contatto ambienti sociali superiori e inferiori, lasciando momentaneamente cadere le barriere della distinzione sociale. Così questo rimatore aristocratico può esaltare un antico vescovo schieratosi contro le antiche istituzioni della gioventù, e vedere nelle intemperanze di quest'ultima gli eccessi di una folla sediziosa, pur arrivandoma per puro omaggio al proprio culto dei fatti locali e delle illustri casate cittadine - a ricopiare dall'annalista cittadino Ghelini l'elenco dei giovani perseveranti nella protesta: membri di famiglie distinte, non della bassa plebe.

Fino al XVIII secolo continuano a essere riproposte misure restrittive o repressive degli usi più turbolenti della gioventù, ormai ritenuti indecenti; tanto più dopo che da tali pratiche sociali si sono ritirati i ceti superiori, mentre l'organizzazione delle ritualità satiriche giovanili si è concentrata nelle mani di elementi dei ceti popolari. Ma nel XIX secolo nessuno si mette più a fare apposite leggi per vietare le scampanate, che dovrebbero essere sanzionate secondo i generici divieti al chiasso notturno e a coercizioni verso singole persone e famiglie. Bollare i partecipanti a questi chiassi come scandalosi perturbatori del riposo notturno e della quiete pubblica è diventato abituale per i ceti superiori; ma in genere senza che si vada oltre una blanda condanna morale, accompagnata da prudente sopportazione, talvolta in un atteggiamento ambiguo tra pubblica riprovazione e privata corrività. Ugualmente, la mobilitazione delle forze di polizia per impedire questi schiamazzi è in molti casi considerata dai tutori dell'ordine una misura sproporzionata, rischiosa e in fin dei conti controproducente. Di fronte alla forza della tradizione, guardie, sindaci e delegati politici preferiscono chiudere gli occhi, o limitarsi a mantenere la situazione sotto controllo senza mostrarsi.

In un susseguirsi di incerti provvedimenti locali, dal XVI al XVII secolo i divieti non restano senza effetto, ma nella maggior parte dei casi rimangono a lungo difficili da applicare; a maggior ragione nelle campagne. Si ricordi che in talune città i primi interventi inibitori risalgono addirittura al XIV e XV secolo. Anche dalle cronache di Alessandria apprendiamo che nel XVI secolo comincia a esaurirsi l'epoca in cui i diritti della scampanata erano trattati quasi alla stregua di una tassa municipale, o di una decima dovuta a una confraternita parrocchiale della gioventù, come appunto avveniva col gioco d'Acheronte. Un processo che si può dire definitivamente concluso solo entro il XVIII secolo, quando la maggior parte delle autorità arriva a ritenere queste pratiche delle estorsioni, e il fracasso che le accompagna - pur codificato da usi tradizionali - un disordine ingiustificato. Altro discorso è però aspettarsi che la prevenzione sia efficace e che ancora nel XIX secolo venga meno completamente la tolleranza verso simili usanze. Ancora a metà del XIX secolo, l'economista genovese Gerolamo Boccardo, nell'elencare 
le tradizioni festive italiane da riformare come nuovi rituali civili, e le sconcezze da vietare invece risolutamente, evoca tra queste ultime il gioco d'Acheronte e pure le asinate, derisioni a persone messe rovesciate a cavalcioni sugli asini: usanze che in qualche regione riapparivano periodicamente. ${ }^{26}$ Perciò le conseguenze delle proibizioni vanno valutate storicamente sia nella breve che nella lunga durata. Occorre valutarle assieme alle generali trasformazioni della mentalità, che porta a un maggiore riconoscimento dell'individuo e delle sue scelte; inoltre al mutare dello spazio pubblico, col progressivo pieno affermarsi dello stato moderno e della sua capillare capacità di controllo sulla società.

\section{Notes}

${ }^{1}$ M. Fincardi, Derisioni notturne. Racconti di serenate alla rovescia (Santa Maria Capua Vetere, Spartaco, 2005).

${ }^{2}$ Hardy già nel suo romanzo The Mayor of Casterbridge (1886) ha ampiamente descritto l'uso sociale dello skimmington con pentole e corni.

${ }^{3}$ S. Clark, 'Inversion, Misrule and the Meaning of Witchcraft', Past and Present, 87 (1980), 98-127; M. Ingram, 'Ridings, Rough Music and the "Reform of popular Culture" in Early Modern England', Past and Present, 105 (1984), 79-113; M. Ingram, 'Le charivari dans I'Angleterre du XVIe et du XVIle siècle', in Le charivari, a cura di J. Le Goff e J.-C. Schmitt (Parigi, La Haye e New York, Mouton-EHESS, 1981), pp. 251-64.

${ }^{4}$ P. Gayrard, '"Monsieur de Pourceaugnac": un charivari à la cour de Louis XIV?', in Le charivari, pp. 309-17.

${ }^{5}$ S. Butler, Hudibras (Oxford, Clarendon Press, 1967), parte II, canto II, vv. 566-68 (pp. 142-49).

${ }^{6}$ T. Folengo, Baldus (Torino, Einaudi, 1989), libro VII, vv. 281-84 (p. 228).

${ }^{7}$ Si veda P. Burke, Popular Culture in Early Modern Europe (Londra, Temple Smith, 1978).

${ }^{8}$ M. Buonarroti, La fiera [1619] (Firenze, Olschki, 1984), scena VIII, vv. 178-79 (p. 60).

${ }^{9}$ G. B. Fagiuoli, Le nozze del diavolo [1729], in Rime piacevoli, 15 voll. (Colle, Pacini, 1827), III, 32-33.

${ }^{10}$ Proveniente da una famiglia di noti letterati umanisti, Forteguerri si firma Carteromonaco, col vezzo di tradurre il proprio cognome in greco, per non esser confuso con I'omonimo cardinale, suo antenato, vissuto quasi tre secoli prima. Da questo suo poema pseudoariostesco Gioacchino
Rossini trarrà un'opera lirica semiseria, Ricciardo e Zoraide, rappresentata per la prima volta nel 1818 al teatro San Carlo di Napoli, poco riuscita a causa del libretto raffazzonato di Francesco Berio di Salsa.

${ }^{11}$ N. Forteguerri, Ricciardetto, edizione critica a cura di C. Di Donna Prencipe (Bologna, Commissione per i testi in lingua, 1989); da ora questa edizione verrà segnata con la lettera $R$, seguita dai numeri riferiti ai canti e alle stanze.

${ }^{12}$ B. Napoli, Dei baccani che si fanno nelle nozze de' vedovi, detti volgarmente cembalate, o scampanate. Dissertazione teologica, e istoricocritica (Lucca, Marescandoli, 1772), p. 51.

${ }^{13}$ F. Ferraironi, I ciaravügli (stamburata) (Roma, Sallustiana, 1951), p. 3. Dalla versione riportata dal monaco è rielaborato il testo riportato nell'Appendice 1.

${ }^{14}$ L. Ricca, Viaggio da Genova a Nizza, 2 voll. (Firenze, Calasanziana, 1871; riedizione anastatica Bologna, Forni, 1972); G. Delfino, 'Usanze nuziali liguri "U ciaravugliu"', Archivio per le tradizioni popolari della Liguria, 5, ii (1975), 42-50; A. Schmuckher, Folklore di Liguria, 3 voll. (Genova, Cassa di risparmio di Genova e Imperia, 1989), I, 142-44. Più attenti al Levante ligure e alla Lunigiana: G. Rezasco, 'Scampanata', Giornale linguistico di archeologia, storia e letteratura, 11, ix-x (1884), 321-35; U. Mazzini, 'Note di folklore lunigianese', Giornale storico della Lunigiana, 9 (1919), 189-99.

${ }^{15}$ Si veda Fincardi, Derisioni notturne.

${ }^{16}$ Si veda M. Agulhon, Pénitents et francs-maçons de I'ancienne Provence (Parigi, Fayard, 1984), pp. 29-64. Più problematico, rispetto alle proibizioni legali e alle 
censure culturali, di cui traccia un quadro più contrastato, meno lineare, è M. Vovelle, Les métamorphoses de la fête en Provence de 1750 à 1820 (Poitiers e Parigi, AubierFlammarion, 1976), pp. 13-54. Si veda inoltre A. Torre, 'La rama di Montaldo', in Charivari: mascherate di vivi e di morti, a cura di F. Castelli (Alessandria, Edizioni dell'Orso, 2004), pp. 125-52.

${ }^{17}$ P. Vayra, 'Attentati contro la libertà del matrimonio', in Curiosità e ricerche di storia subalpina, 5 voll. (Torino, Firenze e Roma, Bocca, 1876), II, 174-85; G. C. Pola Falletti di Villafalletto, Associazioni giovanili e feste antiche, 4 voll. (Milano, Costa, 1943), IV, 449-80, e La juventus attraverso i secoli (Monza, Bocca, 1953), pp. 151-71; R. Corso, 'Capramarito', in Enciclopedia italiana, a cura di E. Treccani (Roma, Istituto dell'Enciclopedia italiana, 1949 [seconda edizione]), VIII, p. 903; F. Cognasso, L'Ittalia nel Rinascimento, 3 voll. (Torino, Utet, 1965), I, 45, 601-3; C. Corrain e P. L. Zampini, Documenti etnografici e folkloristici nei sinodi diocesani italiani (Bologna, Forni, 1970), pp. 205-8.

${ }^{18}$ M. Agulhon, La République au village (Parigi, Seuil, 1979), pp. 159-62; N. Zemon Davis, 'The Reason of Misrule: Youth Groups and Charivaris in SixteenthCentury France', Past and Present, 50 (1971), 41-75; E. P. Thompson, '"Rough Music": le charivari anglais', Annales ESC, 27, ii (1972), 285-312, rivisto e ampliato in E. P. Thompson, Customs in Common: Studies in Traditional Popular Culture (New York, The New Press, 1991), pp. 467-537; R. Bonnain Moerdyk e D. Moerdik, 'À propos du charivari: discours bourgeois et coutumes populaires', Annales ESC, 32, ii (1977), 381-98; E. Weber, Peasants into Frenchmen (Stanford, Stanford University Press, 1976); P. Brunello, Ribelli, questuanti e banditi (Venezia, Marsilio, 1981), pp. 11-13; G. C. Barozzi, 'La pentola e la rivolta', Annali Istituto Alcide Cervi, 5 (1983), 227-50; F. Ramella, Terra e telai (Einaudi, Torino, 1984), pp. 227-37; M. Fincardi, 'La memoria della cioccona in Emilia. Appunti per iniziarne lo studio storico', in Charivari, pp. 153-73 (p. 157); su una permanenza di simili forme di protesta nel XX secolo: P. C. Grimaldi, 'Dal disordine all'ordine. Controllo politico e modelli religiosi in due ciabre di un paese di Langa', in Tempi grassi, tempi magri (Torino, Omega, 1996), pp. 107-21.

19 // Gioco d'Acheronte. Poemetto, manoscritto del XIX secolo conservato presso la Biblioteca Civica di
Alessandria, fondo Poesie di Pietro Gaspare Melazzi, vol. XXI (1834), ms. 142. Ringrazio l'amico Franco Castelli per avermi segnalato quest'opera. Si vedano gli estratti del testo riportati nell'Appendice 2; da ora, le citazioni verrano segnate con la lettera $G$, seguita dai numeri riferiti ai canti e alle stanze.

${ }^{20}$ F. Castelli, 'Charivari, scampanata, ciabra, ovvero il "gioco d'Acheronte"', in Charivari, pp. VII-LXIII (pp. XXIV-XXX). Non è chiaro perché venga chiamato gioco d'Acheronte. Forse si pensava che simulasse un corteo infernale, dal nome del fiume che delimitava dei dannati. Nel XIX secolo, l'economista genovese Gerolamo Boccardo, tra le 'feste e ricreazioni' da vietare, citava come esempio clamoroso 'I'antico giuoco d'Acheronte (in alcuni paesi è detto il giuoco delle Tenebre), nato forse dal pregiudizio sfavorevole, da tempo immemorabile invalso, contro le seconde e terze nozze'. Lo definiva una 'stolta ed immemorabile berlina, [...] che tuttora sussiste in alcune provincie, per esempio nella Liguria Marittima'; G. Boccardo, Feste, giuochi e spettacoli (Genova, Istituto sordomuti, 1874), p. 169.

${ }^{21}$ A. Guasco, Tela cangiante (Milano, Pontio e Piccaglia impressori archiepiscopali, 1605). II clima culturale alessandrino nel riformare i costumi popolari tra XVI e XIX viene ricostruito da F. Castelli, 'Charivari, scampanata, ciabra', pp. XXIV-XXX.

${ }^{22}$ G. Ruffini, Lorenzo Benoni or Passages in the Life of an Italian (Edimburgo e Londra, Constable-Hamilton \& Adams, 1853), pp. 4-5.

${ }^{23}$ Si veda M. Ozouf, Les aveux du roman. Le XIXe siècle entre Ancien Régime et Révolution (Parigi, Gallimard, 2001).

${ }^{24} \mathrm{~F}$. Neri, 'Le abbazie degli stolti in Piemonte nei secoli XV e XVI', Giornale storico della letteratura italiana, 40, nn. 118-19 (1902), 23-35; S. Gini, Note sulle abbazie degli stolti in Piemonte (Bologna, Zanichelli, 1905); G. C. Pola Falletti di Villafalletto, Le gaie compagnie dei giovani del vecchio Piemonte (Casale Monferrato, Miglietta, 1937 [rist. anastatica Torino, Omega, 1995]); P. Scotti, 'Le antiche badie dei giovani', Convivium, 12, iv (1941), 419-29.

${ }^{25}$ Vovelle, Les métamorphoses.

${ }^{26}$ Boccardo, Feste, giuochi e spettacoli, pp. 167-69. 


\section{Appendice I}

\section{Luca Maria Capponi, I Ciaravügli}

1 Una sèira der mese d'uttubre, chietu, chietu m'en stava à fenèscia aspeitòr fusse chóita a menescia, e pöi tra paglia andòrme a slongor,

2 Tütt'assème ün fracassu mi sentu: ün rebúmbu vein d'â Sambüghea; de mazzàmi ena grossa luvéa tütt'assème mi vegu arrivor.

3 Gh'èira â testa ün omu sc'en anze, cun ciümagliu all'antìga ar capé, e a marscina guernia de papé. 'Capitaniu' au sentava ciamor.

4 U marciava d'ün passu cumpertu; u s'enriàva de quandu en quandu; d'â man drita u bastun der cumandu, e d'â lérca tegnava u buccò.

5 Pöi, cun vuxe d'ún ton segrurusu, u ragliava: - Figliöi, stèi attenti: tegnì prunti i vössci strumenti, e mettèive a ciaravüglior.

6 Un fracassu ar mundu parescu, ün bisbigliu, ün disurdine eguale, creèi püre, mi nu sentu p'u quale, difficile purerlu spiegor.

7 Peggiu assoi che de crave ün varíe, quand'i sente: 'sò, sò' ch'ün ghe dixe, s'arrabata piccúin e gambíxe, ai sarin sen van arrugior.

8 Lì gh'è cörni, tamburdi, tabassi; lì chi süggia, là chi caramella; chi picunra sci'ün testu o paella; e va chi pó ciü bein sciarattor.

9 Chi stirazza ün ancüge, ün perfèrru, chi u sbrandò, chi i trempèi, chi a caénra, marapicche de qualunque menra; fin cheicün cu l'avia u tagliavú.

$10 \mathrm{Mi}$, curiusu che sun sempre stau, de Frumé dagu man â sonaglia; m'accumpagnu cun tütta a canaglia per saver cume a va a terminor.

1 Una sera del mese d'ottobre, quieto quieto stavo al balcone aspettando fosse cotta la minestra, per andarmene poi ad allungarmi sul pagliericcio.

2 D'un tratto sento un fracasso: un rimbombo vien dalla Sambughéa; ${ }^{1}$ di ragazzaglia c'è una gran gazzarra a un tratto lo vedo arrivare.

3 C'era in testa un uomo su un asino, con pennacchio all'antica al cappello, la marsina guarnita di carta.

'Capitano' lo sentivo chiamare.

4 Marciava con passo solenne; sorrideva di quando in quando; nella mano destra il bastone del comando, nell'altra teneva la cavezza.

5 Poi con voce di tono cavernoso arringava: - Ragazzi, state attenti: tenete pronti i vostri strumenti, e mettetevi a ciaravugliare.

6 Un fracasso al mondo simile, un sibilo, un disordine uguale, siate certi, lo dico cosciente, è difficile poterlo spiegare.

7 Peggio assai d'un branco di capre quando sente chiamar: 'sale, sale', che s'avventa, campàni e collari agitando, a1la piastra del sale si precipitano.

8 Ci son corni, grancasse e tamburi; lì chi fischia, là chi ciaramella; c'è chi batte su testi e padelle; e si fa a chi più fa baccano.

9 Chi trascina un incudine o un vomere, chi l'alàre, i treppiedi o catena; ${ }^{2}$ vecchi picconi di ogni tipo; qualcuno batteva fin il tagliere.

10 lo, curioso che son sempre stato, di Frumé ${ }^{3}$ do mano alla sonagliera; m'intruppo con tutta la canaglia, per saper come va a finire. 
11 Arrivòi att carruggiu Suttan, ferma l'anze e aissa u bastun:

'Addè è tempu: custanza e uniun'. E sebúto $\mathrm{m}^{\prime}$ han foitu assavor.

12 Se presenta a Giousè de Brazzin e u ghe dixe: 'Giousè ve salüu; savé bein perché mi sun vegnüu: via, Giousè, nu ve féi cugliunor.

13 Sevé viduu, e üna vidua spusei; savé bein l'üsanza ch'a l'è chì'. Mi ghe digu: cusci fusse mi! Me respunde: - Franzé, nu parlor.

14 Capitano - Mi ghe sun già passau cume vui: ve renunziu u bastun der cumandu. Glie dii pocu? Ma sulu, ma quandu n'averé bein sapüu cuntentor.

15 Che se pöi vögliessèi for de testa; che se pöi vögliessèi for de düru, ve prumettu, Giousè, v'assegüru che per ün mesu n'avé da chietor.

16 E per megliu cumpíe sta facenda, zercheremu un ber anze rüscáu; voutu â cua gh'aisseremu u spusau, per Triöra au faremu giror.

17 L'è pan resu: m'han fòitu parescu. Nui vuremu tratorve da amighi: mantegne l'úsu d'ii tempi antìghi; ma, Giousè, vui v'avé da bagnor.

18 Vedovo. - Mi capisciu che vèin che me bagne; via, dîsciü; che pretendei voi? Ma ve pregu d'üsor caritòi; sagu bein nu vurré me spellor.

19 Cap. - Nui saremu ancor troppu discretti; ma tre seglie de vin de Gerbónte procurei che bein cenre sie pronte; e üna mina de fighe a ghe vor.

20 Che dî vui, Franzé, che ve por? Franzé. - Nu ve séve levati da l'onestu. Ved. - Sun d'accordu e men vagu, bein prestu zo ch'avé ditu a fòrlu portor.

21 Capit. - E vui autri, mei camerada, per dòrme ün segnu d'esse cuntenti, dèi, sciü, de man ai vösci strumenti, turnéi ün pocu a ciaruvüglior.
11 Arrivati al carruggio Sottana, [il capitano] ferma il ciuco e alza il bastone:

'Andate a tempo: costanza e unione'.

E subito $\mathrm{m}^{\prime}$ han fatto tacere.

12 Si presenta [il Capitano] a Giuseppe dei Brazzin ${ }^{4}$ e gli dice: 'Giuseppe vi saluto; sapete bene perché son venuto:

Su, Giuseppe, non vi fate sberteggiare.

13 Siete vedovo e sposate una vedova; sapete bene l'usanza che c'è qui'. lo gli dico: 'Così fossi anch'io' [cioè vedovo risposato]. Mi risponde: 'Franzé, non parlare'.

14 Capitano - 'Ci son già passato anch'io come voi: vi cedo il bastone del comando. Vi par poco? Ma solo, ma quando voi ci avrete ben contentati.

15 Che se poi voleste fare un colpo di testa; che se poi voleste ostinarvi, vi prometto, Giuseppe, vi assicuro che per un mese non avreste quiete.

16 E per meglio compiere la faccenda, cercheremmo un bell'asino piagato; voltato alla coda ci metteremmo sopra lo sposo, lo faremmo girare per Triora.

17 È pan reso: m'han fatto allo stesso modo a me. Noi vorremmo trattarvi da amici: mantener I'uso dei tempi antichi; ma, Giuseppe, voi dovete sborsare.

18 // vedovo - Capisco che bisogna che paghi; ovvia, dite, su: che pretendete? Ma vi prego usatemi carità, so bene che non vorrete pelarmi.

19 /l Capitano - Noi saremo anche troppo discreti; ma tre secchi di vino di Gerbónte ${ }^{6}$ procurate che siano pronti, e ci vuole una mina ${ }^{7}$ di fichi.

20 - Che ne dite, Franzé, che vi sembra? Franzé - Vi siete mostrati giusti. II vedovo - Son d'accordo e vado, ben presto ciò che avete chiesto lo farò portare.

21 /l Capitano - E voi altri, miei camerati, per mostrargli un segno che siete contenti, dai, su, date mano ai vostri strumenti tornate un po' a ciaravugliare. 
22 Franzé. - Magineive che sghiggi, che treatu, Ma, addè addè, u l'è chì u beveragiu; u l'arriva, fornisce u tapagiu; pressu e fighe sen van arrulor,

23 Quer de l'anze u l'è u primu: gh'attocca; u fa ün sbrindu a Giousè, a l'üsanza; e per partie cun bona creanza sen va de tira a delicenzior.

24 Capit. - Via, Giousè - u ghe dixe riendu marieive; bon pru; mi ve lasciu; ma guardei che per vui u l'è ün fasciu, e nu soi se u purré ciü portor.

25 Ved. - Ve rengraziu, respunde u spusau; n'hoi pieixé che siei tütti cuntenti; ma, per parlorve fora dei dénti, entru restu n'avé da pensor.

26 Franzé. - I autri entantu nu fan zerimonrie; n'han besögnu de die ch'ün se sbrighe; cume luvi i se raspa ste fighe, e cun a cuppa i tende a lapor.

27 Quandu tüttu fu a reu nesteggiau, cume a föglia che u ventu sparpaglia, scapa via tütta quanta a canaglia. E cuscì a festa andò a terminor.

\section{Note}

1 Sambughéa: contrada del rione Camurata nella zona bassa di Triora.

2 /'alàre, i treppiedi o catena: tutti accessori del camino.

${ }^{3}$ Frumé: nome da vacca.

${ }^{4}$ Brazzin: soprannome di famiglia.

5 'Cosi fossi anch'io': cioè vedovo risposato.

${ }^{6}$ Gerbónte: frazione, a mille metri di altitudine, dove i Brazzin avevano il vigneto.

${ }^{7}$ mina: staio da 10 litri.

${ }^{8}$ Quello dell'asino: il capitano.
22 Franzé - Immaginatevi che urli, che fracasso. Ma or ora è qui il beveraggio; giusto appena arriva, finisce il fracasso; presso i fichi si vanno a fermare.

23 Quello dell'asino ${ }^{8}$ è il primo: gli spetta; sollecita un brindisi a Giuseppe, come d'uso; e per partire con buona creanza si fa subito parte e si congeda.

24 // Capitano - Orsù, Giuseppe - gli dice ridendo maritatevi; buona fortuna; vi lascio; ma badate che per voi è un bel peso, io non so se potrete reggerlo.

25 /l vedovo - Vi ringrazio, risponde lo sposo; n'ho piacere che siate tutti contenti; ma, per parlarvi fuori dai denti, per il resto non dovete pensarci.

26 Franzé - Tutti gli altri intanto non fanno cerimonie; non occorre dire che si sbrighino; come lupi arraffano i fichi, e con la coppa sbevazzano.

27 Quando tutto è sparito nel gozzo, come le foglie che il vento sparpaglia, scappa via tutta la canaglia. E così la festa giunse al termine.

(Testo citato da F. Ferraironi, I ciaravügli (stamburata) (Roma, Sallustiana, 1951), pp. 9-14; traduzione di Marco Fincardi) 


\section{Appendice II}

\section{Pietro Gaspare Melazzi, II gioco d'Acheronte. Poemetto (1834)}

\section{[Dal canto I]}

1 Non invochiam più Apollo, né le suore Che abitavan un di sul Sacro Monte. Le discacciaro nel più cupo orrore I Romantici, che hanno ai cirri pronte Le Tempeste, le Nebbie, e i Temporali, Che nè pochi Lor sono immortali.

2 Ma come necessari i Numi sono A quel che imprende una qualunque impresa Così m'invocherò Turbine, o Tuono,

E se la fantasia feconda non resa Di tanti Autori dell'età presente, Così pur d'estro accenderan mia mente.

3 E il Tema che ho a trattare ha sol che fare Con cose che arricciar fanno la fronte. Egli era questo sprezzo singolare Ed era detto Gioco d'Acheronte: Ed era veramente una perfidia Degna d'Averno, o almeno di Numidia. [...]

\section{[Dal canto II]}

$5 \quad[\ldots]$

E descriviamo adesso l'uso antico che di seconde nozze era nemico.

6. Chi voleva in allor rimaritarsi pagar doveva a un certo qual Prefetto una multa in danaro da impiegarsi in parte per il Santo benedetto ch'era di quella corte il protettore e l'altra la pappava quel Signore.

7. E guai a quella Cara Vedovetta che la multa pagar si rifiutava! Allora si suonava la trombetta e il Gioco d'Acheronte si annunziava il quale or a descriver intraprendo che già di sempre compiacervi intendo.

8. E lo stendardo fuor del suo soggiorno allor si traeva: in lui sedente v'era una Capra, ed asinelli intorno dipinti da un Pittor sommo valente, e molte vecchie v'eran che filavano e la Capra cornuta rimiravano.

9 Questo stendardo molt'anni posò nella nobil famiglia degli Bianchi e in quella poi degli Olio trapassò, e quando questi fur di quello stanchi i Bianchi nuovamente lo pigliaro e per molt'anni poi lo conservaro.

10 Mentre il Ghilini i suoi scriveva annali questo stendardo ancora si vedeva e si portò nei dì di Carnevale quando la mascherata si faceva ma il tempo che distrugge ohimè! pur tutto ha pure il mio stendardo ancor distrutto.

11 I Modenesi almen son da lodarsi, da lor la secchia si conserva ancora. È vero, che per Lei dovè pugnarsi $\mathrm{E}$ che si sparse molto sangue allora Ed il Tassoni ci lasciò memoria Di questa rara interessante istoria.

12 Disposto pur vi stava un Asinello bardato in tutta pompa, e nastri e fiori ornavan le sue orecchie in modo bello, come si soglion adornar gli Amori... ma in vero il paragone alto è un po' troppo, e forse in farlo ho preso or il galoppo.

13 Appena dunque lo stendardo fuori tutta la Gioventù si radunava (ma quella che si aveva maggiori ardori che la tranquilla sempre in casa stava) nel tetto più vicino della sposa che di tornar a nozze era smaniosa.

$14 \mathrm{E}$ allora che la vedova sortiva per gire in chiesa a pronunziare il si la Gioventù dalla casa usciva collo stendardo, e si piantava lì, e l'asinello pur suo menava che per la nuova sposa destinava.

15 E se poi riuscivan nell'intento a rovescio su quello la sedevano, e per accrescer il divertimento la coda in man di quella allor mettevano 
e per le vie della Città maggiori menavanla facendo alti rumori.

16 Cantavan pur sciocchissime Canzoni ch'eran tutte alle Vedove un insulto. Le comparavan esse a quei tronconi ripullulanti fuor qualche virgulto, e che ben tosto cadono in ruina cadendo sovra lor tempesta, o brina.

17 La ricoprivan poi di vituperi gittando polve, o mota sovra Lei talor con atti inverecondi, e fieri stracciavan le vesti di costei, e a rinserrarsi la stringevan poi in angol della casa con i suoi:

18 E per tre giorni pur consecutivi in quella casa stavan tumultuando quasi di senno, e di ragione privi andavan bevendo, e gavvazzando, e mascherati opravano da pazzi, e ciò chiamavan leciti solazzi.

19 Guai se la donna poi sortir dovea per qualche affare della sua famiglia, allora cose orribili vedea:

la faccia le facevano vermiglia, o l'anneravan, come l'hanno quelli, che tolgon la caligine ai fornelli. [...]

21 Es'incontravan qualche buon villano che avesse un qualche carico di legno e l'asinello conducesse a mano allor la gioventù con atto indegno toglierà quella soma, e vi ponea la Vedova, che a spasso conducea.

22 Ciò non pertanto all'Isabella mia Recar puote il minimo spavento Tanto amoroso ardore in sé sentia Di render il suo bel Zampier contento, che il giorno per le nozze ha stabilito ed ai parenti vuol mandar l'invito.

23 La Casa di Costei come vi ho detto è nel quartier di Rovereto antico ed è propinqua di Zampier al tetto, giovinetto distinto, e solo amico di quei che di virtù seguon la via bramando sol la loro compagnia. [...]
37 E dove abbiam lasciata I'Isabella? Il giorno delle nozze ha già fissato Ed in secreto già i parenti appella, celandolo per altro al vicinato, onde schivare tutti i scherni, e l'onte che suol produrre il Gioco d'Acheronte.

38 Ma un'empia strega invero maledetta Svelato aveva tutto il gran mistero, sicché la Gioventù tutta s'affretta per compiere il fissato suo pensiero [...].

\section{[Dal canto III]}

[...]

3 Cotesta nelle notti se ne giva ma nelle notti sol dei Venerdi sotto le noci in grande comitiva a danze oscene fino al nuovo di e coi Demoni, che con Lei danzavano misteri orrendi sempre speravano.

4 Esempli grazia, se nel Ciel splendeva La Luna con i suoi raggi d'argento Certi incantesimi quella si faceva Per cui s'ottenebrava in sul momento O si faceva insanguinata, e rossa Come da mano portentosa scossa.

5 Evocava de spettri essa talora, che l'avello lasciavano atterriti e fra cotesti ve ne furo ancora di Vedove, che insulti avean patiti, e sofferte le infamie, e i scorni, e l'onte dell'usitato Gioco d'Acheronte.

6 Ebben cotesta strega, che col diavolo Tenea commercio continuato, e stabile, e che di fede non aveva un cavolo penetrò nel mistero impenetrabile, ed avvertì quei Giovin, che Isabella all'Alba maritavasi novella.

7 Sicché si radunaro tutti quelli sulla Piazzetta detta Monferrato che allora appartenevasi alli Marchelli, adesso estinto nobile Casato, e si nascoser tutti all'altrui sguardo dietro il tetto di quel collo stendardo. [... ] 
9 E l'altro scaldatissimo drappello nell'angiporto entrò dei De Amici e s'appiattò colà con l'asinello già destinato ai suoi beffardi uffici, la Vedova aspettando, che lasciasse la Casa, ed alla Chiesa se ne andasse.

10 Ma la prudente Vedovella pria D'uscir di casa intorno a sé chiamò Tutta quella ridente Compagnia, e in questo modo quindi favellò... vedete tutti, che può fare Amore, di chi seguace gli è, saggio Oratore. [...]

13 'Cari Parenti, che mi state intorno, e che volete accompagnarmi in Chiesa sappiate che all'uscir dal mio soggiorno da certi scapestrati io sono attesa e preparato d'Acheronte il Gioco: ma di ciò, Cari, me n'importa poco.

14 Rimaritarsi, tutti il sanno, lice: e la legge divina, ed anche umana a tutti tutti apertamente il dice. Onde è che noi dobbiam rendere vana La tracotanza di costor, che stanno Li fuori pronti, onde recarmi affanno.

15 Andiamo in Chiesa, combattiam se è d'uopo, e rintuzziamo il loro perverso ardire| questa è un'usanza che funesto ha scopo... alfin dovesse ella per me finire! Andiamo, Amici: confidiamo in Dio: è giusto, è santo egli è il disegno mio.'

16 Ed ecco I'Isabella in pompa magna Che sorte dalla Casa!... Eccola in strada! Gran turba di Parenti l'accompagna Pronti ad usar, e senno, e mano, e spada, e tra questi i fratelli di Zampiero uno dei quali porta alto cimiero.

17 Egli ad imprese belliche trovossi, e combatté contro Amurat Sultano, e fu tra gli distinti, e gli promossi, e più d'un turco fece a brano, a brano; sicché cercava ben nuova occasione per dimostrarsi un uom di distinzione.

18 Giunge la Sposa appena alla Piazzetta che già vi nominai di Monferrato, che la nascosta Gioventù s'affretta di metterla sull'Asin preparato

e il Banderajo là pur si ritrova

con chi vuol tentare la brutta prova.

19 Ma quest'osso a mangiarsi è molto duro; diffendesi la Sposa dai Parenti, e posta essa in un luogo assai sicuro combattono costor da prodi, e ardenti, e quella Gioventù dispersa viene, come da venti le più asciutte arene.

20 Ciò fatto la Sposina lietamente siegue con tutti l'intrapresa via, e vuol saper chi è stato il più valente in rintuzzar la perfida genìa, e sente con piacer, che fu il fratello del caro amato suo sposo novello.

21 E tosto il fa chiamar, volendo ad esso mostrar, che nutre un cor riconoscente, e in ogni modo che le vien concesso gli mostra gratitudine, e repente si toglie un bel anello dalle dita, e glielo da con grazia in ver compita.

22 La Comitiva tutta applaude allora A questo tratto bel di gentilezza... Ma intanto in chiesa sono giunti ancora Ove di tutto già si avea contezza, e d'onde avean mandato d'ogni affare il vescovo Guarnero ad informare. [...]

26 Ed Isabella allor lieta risente Quello, che il Celebrante le ridice Cioè che possa viver lungamente E possa sempre ella essere felice, e possa rimirare i Pronipoti e dice nel suo cuor... Che cari voti!...

27 Ma mentre la funzion si compie in Chiesa La Gioventù in numero cresciuta In sulla Piazza dei Moroni accesa di rabbia, e sdegno allor tutta è venuta, gridando ad alta voce: questa volta la Vedovella a noi non sarà tolta.

28 Fondati eran Costor sul detto antico, che gli audaci protegge la Fortuna: e non pensavan poi, che un Nume amico vi è pur, che faccia quella nube bruna, che di grandine minaccia, ovver rovina a chi solo a Virtù si prostra, e inchina. 
29 Intanto Monsignor Guernero [sic] Trotti Intorno a sé adunati in fretta in fretta I sacerdoti più eloquenti, e dotti, d'andar con Essi subito si affretta a Santa Maria detta del Castello, ove succeder poteva un macello. [...]

\section{[Dal canto IV]}

1 Quell'insolente Gioventù schiamazza Credendosi sicura dell'intento, e quasi occupa tutta quella piazza, ed il di Lei Prefetto a grave stento ottiene, che non entri essa nel Tempio... Sacrilego sarebbe il tratto, ed empio,

2 E ad alta voce grida: fuori, fuori La Sposa: la porrem sull'Asinello, e noi la cingerem di vaghi fiori: vedrem se salveralla quel Drappello, che la salvò testè: mostri coraggio, ed ora schivi il meritato omaggio.

3 Vedrem se basteranno i suoi parenti, ed il fratello del Sig. Zampiero, che credesi capace di portenti; perché porta sul capo alto il cimiero: vedrem se basterà quegli per trarla or dalle nostre mani: e risalvarla.

4 Era il mattino allor che ciò avveniva Giunto ad un'ora più di sua metà [...].

6 Mentre in tai modi tumultuando vanno... dalla Chiesetta sacra al Santo Legno su cui moriva con immenso affanno chi di Satanna debellava il regno, spuntò con i suoi preti Monsignore, e tutti egli calmò d'alto terrore.

7 E a quei rivolto con il volto austero in alto fulminante un anatema gridò: quale nutrite ora pensiero? Sgombrate questi luoghi... che non si trema O scapestrata Gioventù d'offendere Quel che vi puote con un cenno incendere.

8 Ritorni ognuno a casa tostamente, ed annientate quel fatal stendardo. . se ritornar a Nozze si consente da Chi tiene su Voi, su me lo sguardo, perché volete a Quel Voi fare insulto ed insultar pur anco il Cristian culto?

9 Se mai scrit[t]o fosse a questi dì di trar un paragone dalla Storia direi, che da Calcante s'atterrì nessuno mai così, di chi con gloria pugnava contro d'llio con speranza di vincere dei Teucri la costanza.

10 A questi detti dunque incominciaro a sparpagliarsi i ragunati allora, e solo sulla piazza si restaro alcuni pochi pertinaci ancora, e il Bianchi, che portava lo stendardo a andarsene di là non fu il più tardo.

11 Alcuni dei rimasti io notar voglio restovvi Falameri, e Bertalocco, Milani, Pettenari, e Briaroglio, Pinazzo, Squarzafichi con Majocco, e Spelta, e Tavernaro, ed Engerloni, Fornari, Caresani, e due Ardizzoni.

12 || Trotti intanto visto che ottenuto avea l'intento in parte, in Chiesa entrò, e ai Sposi per offrir maggiore ajuto con chi lieto allor s'accompagnò, e i Preti il seguitar fin dove detto Zampiero aveva per la Sposa il tetto.

13 Quei pochi giovinastri ancor fremevano, che sopra quella piazza eran rimasti, perché sfuggita a loro si vedevano la preda per cui fatti avean contrasti. Ma il fremer lor simile affatto egli era A quel del can a Cingia in notte nera.

14 Con tutti i preti puoi fu Monsignore trattato con rinfreschi, e ringraziato. Tutti studiar di fargli quell'onore, che si era veramente meritato, e voglio anch'io tentar d'accrescer gloria a me con far di Lui grata memoria. [...]

16 Era Guernero [sic] Trotti di famiglia oriunda di Milano, e in vero antica. Ma in Alessandria aveva al dì le ciglia Aperte; e un'alma avea sortita amica Sol di virtude, ed al ben fare inclina, ed acquistata avea somma dottrina. [...] 
18 Egli non era come Adam Boschetto, che vescovo era un dì dei Giminiani, il quale era in pochissimo concetto e non aveva mai l'ufficio in mani e in cambio di dir vespre, e mattutino, giocava tutto il giorno a sbaragliano.

19 Invece egli per tempo lì c'estava, e in ogni dì leggeva la Scrittura forte, da cui le massime pigliava per trar il Gregge al ben, che aveva in cura, il che servir dovrebbe ora d'esempio a chi porta la Mitra entro nel Tempio.

20 Ebben costui non pago d'aver reso I'empio disegno lor vuoto d'effetto, egli un affar maggior ave intrapreso: il Gioco d'Acheronte egli ha interdetto, e il fece pur vietar dal Podestà che amministrava allor questa Città.

21 E lo era un Cadamosti lodigiano uomo sapiente, veramente retto quanto lo fu quel savio Giustiniano, il di cui Nome è ancora benedetto, perché raccoglier fé le leggi tutte, che senza Lui sarebbero distrutte.

22 E questo Gioco antico si facea per molti già ottenuti privilegi, e in pergamene scritti egli ne avea

per man di Duchi, Imperatori e Regi.

Eppure egli operò sì santamente, che il fece proibir subitamente.

23 Cotesto infame Gioco d'Acheronte Di discordie, di risse, e di dissidii Fu sempre - ohi troppo! - innesauribil fonte; e qualche volta pure d'omicidii, e se ne legge ancor trista memoria in molte carte della nostra Istoria.

24 Eterna gloria dunque a questo Grande che non avea dei Tempi i pregiudizii, e che le usanze solite, e nefande sapeva calpestare al par dei vizii, e che nutriva solo nel pensiero di compiere il suo santo Ministero.

25. Eternamente il nome suo si onori, e il segnano color che eletti sono ad essere dei Popoli Pastori: né mai ponghin sue gesta in abbandono, ch'esser il nome suo scolpito merta in pario marmo da una mano esperta.

(I/ Gioco d'Acheronte. Poemetto, manoscritto del XIX secolo, Biblioteca Civica di Alessandria, fondo Poesie di Pietro Gaspare Melazzi, vol. XXI (1834), ms. 142)

\section{Please address correspondence to: Marco Fincardi, Dipartimento di Studi Storici, Università 'Ca' Foscari', Venezia, Italy}

(C) Department of Italian Studies, University of Reading and Department of Italian, University of Cambridge 